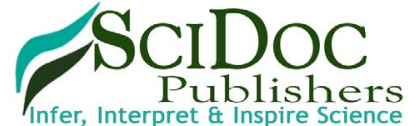

International Journal of Respiratory Disease, Care \& Medicine (IJRDM)

ISSN: 2577-4409

\title{
COPD and Dyspnea: Management of a Severely Affected Elderly Patient (Effect of Comprehensive Directed Breathing during Bi-level Exercises)
}

Gimenez $\mathrm{M}^{1^{*}}$, Lantarón-Caeiro EM ${ }^{2}$, Aranda A ${ }^{1}$, Gómez A Jr ${ }^{1}$, Lorenzo Villalba $\mathrm{N}^{3}$, Bach $\mathrm{JR}^{4}$

${ }^{1}$ Departments of Physical Medicine and Rehabilitation and Respiratory Physiotherapy, University Hospital of Gran Canaria Dr. Negrín, Las Palmas de Gran Canaria, Spain.

${ }^{2}$ Faculty of Physiotherapy Faculty of Physiotherapy - Campus of Pontevedra, Pontevedra, University of Vigo, Spain.

${ }^{3}$ Service de Médecine Interne. Centre Hospitalier Chrétien. Belgium.

${ }^{4}$ Department of Physical Medicine and Rehabilitation, New Jersey Medical School, Rutgers University, USA.

\section{Abstract}

\begin{abstract}
This report is about an eleven year follow up of a 70-year-old man (185 cm tall, $98 \mathrm{~kg}$ of weight) with severe COPD diagnosed in 2006: He was given usual medical treatment, periodically, with bronchodilators, steroids and standard respiratory physiotherapy.

Objective: To compare the effects of Traditional Diaphragmatic Breathing (TDB), and training exercises with those of the association of Comprehensive Directed Breathing (CDB) used at rest and during the 45 minute Bi-levels: Square Wave Endurance Exercise Test (SWEET) training on cycle ergometry, Two Step Stool (TSST) and walking.

Design: The intervention for the two programs (TDB and CDB) included diaphragmatic breathing exercises, cycle ergometry, walk and bi-level stairs sessions for 90 minutes five days/wk. In addition our team taught and explained to the patient, the anatomical aspects, and the physiology of ventilation along with CDB.

The CDB was taught with all the aspects of anatomy and physiology of ventilation; The patient observed his ventilatory dyssynchronism in a mirror; he was shown his ventilatory rhythm on a spirogram; diaphragmatic movement was demonstrated in an educational movie, and verbal feedback was used to correct respiratory asynchrony.

Results: In the first (TDB) traditional treatment, all medical and physiotherapeutic interventions did not effectively improve his dyspnea neither his exercise tolerance. On the contrary, the second (CDB) treatment with SWEET-CDB showed significant improvements in endurance exercise capacity (6-MWT $+30 \%$; SWEET, kJ $/ \mathrm{kg}+63 \%, \mathrm{p}<0.01$; Ventilatory Anaerobic Threshold $+76 \%$; PWR $+30 \%$, in perception of dyspnea (Borg; VAT $=-91 \%$; 120W $-52 \%$; PWR $-46 \%, p<0.01$ ), while $\left(\mathrm{PEmax}=+33 \%\right.$; PImax $=+26 \%, \mathrm{MVV}=+14 \%$, VE $\max =+31 \%$, VT and $\mathrm{O}_{2} \mathrm{P}$ all increased significantly $(\mathrm{p}<0.01)$; in addition, overbreathing as well as $\mathrm{RR}, \dot{\mathrm{V}} \mathrm{CO}_{2}, \mathrm{VO}_{2}$ and $\mathrm{HR}$ were reduced $(\mathrm{p}<0.01)$.

In summary: SWEET- CDB training proved effective because it significantly reduced both dyspnea at rest and during exercises. CDB reduced ventilatory asynchrony, overbreathing, breathing frequency, metabolism, and improved arterial blood gases and ventilatory efficiency.
\end{abstract}

Abbreviations: COPD: Chronic Obstructive Pulmonary Disease; TDB: Traditional Diaphragmatic Breathing; PLB: Pursed Lip Breathing; CDB: Comprehensive Directed Breathing; SWEET: Square Wave Endurance Exercise Test; TSST: Two Step Stool; TMW: Total Mechanical Work; MTP: Maximal Tolerated Power; ECSC: European Community for Coal and Steel; MWT: Minute Walk Test; HR: Heart Rate; MTP: Maximal Tolerated Power; VAT: Ventilatory Anaerobic Threshold.

\section{Introduction}

This is an 11 year follow up of a 70 -year-old man $(185 \mathrm{~cm}, 98$ $\mathrm{kg}$ ) who was diagnosed with severe COPD in 2006: FEV1 $0.8 \mathrm{~L}$ (24\%), and a FEV1/FVC ratio of 28.3\%) [1-3]. A thoracic scanner showed advanced diffuse emphysematous lesions.

\section{Clinical History}

He complained of 15 years of progressively increasing exertional dyspnea (ED) [5-7] and in 2005, COPD was diagnosed. An incremental exercise test (30W/3 min) was performed by cycle ergometry along with electrocardiographic and arterial blood pressure

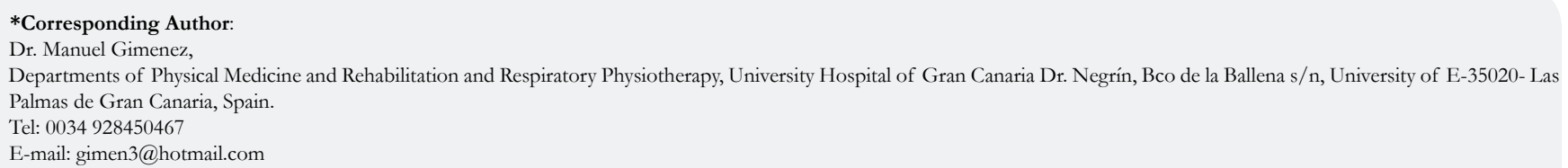

Citation: Gimenez M, Lantarón-Caeiro EM, Aranda A, Gómez A Jr, Lorenzo Villalba N, Bach JR. COPD and Dyspnea: Management of a severely affected Elderly patient. (Effect of Comprehensive Directed Breathing during Bi-level Exercises). 2018; Int J Resp Dis Care Med. 3(1):48-53. doi: http://dx.doi.org/10.19070/2577-4409-180008

Copyright: Gimenez $\mathbf{M}^{\odot}$ 2018. This is an open-access article distributed under the terms of the Creative Commons Attribution License, which permits unrestricted use, distribution and reproduction in any medium, provided the original author and source are credited. 
monitoring [8-11]. The test was suspended after eight minutes and 20s, due to severe exertional dyspnea (ED, 8/9) [7] and 7/8 for leg pain [7]. The $\mathrm{VO}_{2}$ peak [4] was $1.33 \mathrm{~L} / \mathrm{min}$ or $14 \mathrm{~mL} /$ $\mathrm{kg} / \mathrm{min}$. From a second constant $40 \mathrm{~W}$ cycle ergometry during $10 \mathrm{~min}$, a decrease in peripheral oxyhemoglobin saturation $\left(\mathrm{SpO}_{2}\right.$ $=86 \%$ ) was observed, with increasing arterial pressure of $\mathrm{CO}_{2}$ $\left(\mathrm{PaCO}_{2}\right.$ ) (Figure 1). Consequently, the diagnosis was COPD and "alveolar hypoventilation" with hypoxemia and hypercapnia.

Regular medical treatment was given, periodically, with bronchodilators and steroids, and standard respiratory physiotherapy with pursed lips breathing, and low muscular training recommended for a period of three months ( 5 sessions/week). He was advised to breathe with pursed lips and pedal for 30 minutes of cycle ergometry, with very low mechanical resistance. This was well tolerated with little professional monitoring. After this first treatment, despite subjective improvement this did not translate into any clinical improvements, but rather deterioration of dyspnea.

On 02/19/2009, he began our treatment protocol in our respiratory rehabilitation department.

He was no longer able to run more than 50 meters due to intense dyspnea. Therefore, there was a significant reduction of physical activity. This seemed to contribute to weight gain, which in turn increased dyspnea when walking. dyspnea was accompanied by anxiety and paradoxical ventilation. There was no cough, expectoration or edema of lower limbs.

\section{Clinical examination}

The inspection of the facial skin showed a grayish color, cyanosis of the lips and had a moderate deviation of the nasal septum.
Rapid nasal inspiration caused a collapse of the external wall of the left nostril. Thoracic percussion showed increased tympani. Pulmonary auscultation revealed a reduction of the vesicular murmur. The respiratory rate was high (about 28 breathes/min, as measured by auscultation). Cardiac auscultation was normal. Systemic blood pressure was $140 / 80 \mathrm{mmHg}$, with tachycardia after 15 minutes at rest: 96 beats $/ \mathrm{min}$.

\section{Muscle exercise}

Four exercises were performed, which were monitored and recorded by the physiotherapist and the physiatrist:

- Six-minute walk test (6-MWT [8]): 385 meters. $\mathrm{SpO}_{2} 93 \%$ at rest, and the first minute. The successive minutes up to the sixth: 88 , $87,88,88$, and $86 \%$ respectively. Heart rate (HR): 121 beats/min in the sixth minute. Therefore, hypoxemia was noted during the full six-minute test.

- "Twostepstool" Test (TSST [10]) with two $15 \mathrm{~cm}$ high steps: The subject went up and down (1 trip) as many times as possible until tired, then dyspnea occurred after 1 min and 30s: the HR was then 117 beats / min and SpO2 decreased to 89\% (see Table 1).

- $40 \mathrm{~W}$ Constant exercise [4] during 10 minutes, followed by the incremental cycle ergometry exercise test $30 \mathrm{~W} / 3 \mathrm{~min}$ up to the maximum power held for three full minutes [4] reaching $60 \mathrm{~W}$ with HR of 132 beats / min and $\mathrm{SpO}_{2}$ dropped considerably to $76 \%$.

- Square Wave Endurance Exercise Training (SWEET) [11-13] for 45 minutes [(Base 20W x 4 min/60W Peak x $1 \mathrm{~min}$ ), (see above table )]: at $45^{\text {th }}$ min $\mathrm{SpO}_{2} 90 \%$ and HR 124 beats $/ \mathrm{min}$. This test was removed and then performed with oxygen therapy with $\mathrm{SpO}_{2}>$ $94 \%$, just after the 10 th session of the first initial muscular training (Table 1) [11, 12].

Figure 1. shows several physiological facts before and after learning Comprehensive Directed Breathing (CDB). First, the global ventilation; the ventilatory frequency, oxygen consumption and $\mathrm{PaCO}_{2}$ are all significantly elevated while the Tidal Volume (VT) and the $\mathrm{O}_{2}$ saturation are decreased. On the other hand, after application of the CDB, the scenario changes for equivalent global ventilation: $\mathrm{VO}_{2}$, ventilatory frequency, Tidal Volume and $\mathrm{PaCO}_{2}$ are significantly lowered and close to the standard of normal values, while the $\mathrm{SaO}_{2}$ increases approaching the normality of healthy subjects of the same age.

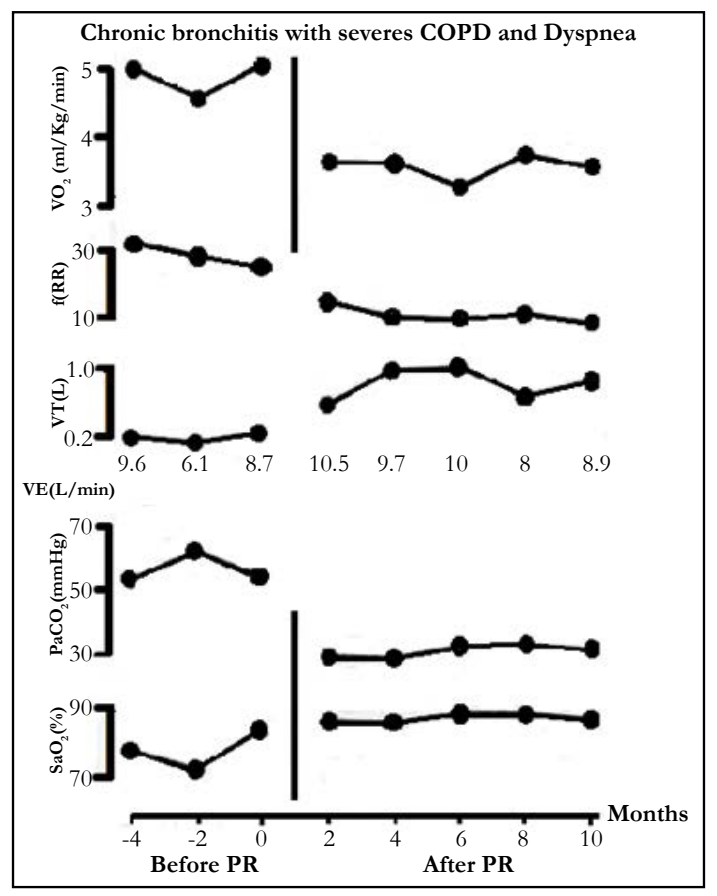


Table 1. SWEET training levels evolution (at Base and Peak) in a patient with severe COPD and Dyspnea.

\begin{tabular}{|c|c|c|c|c|c|c|c|c|c|c|c|}
\hline Date $(*)$ & $2 / 19 / 2009$ & $2 / 20 / 2009$ & $2 / 23 / 2009$ & $2 / 25 / 09 a$ & $2 / 25 / 09 b$ & $2 / 26 / 2009$ & $2 / 27 / 2009$ & $3 / 2 / 2009$ & $3 / 3 / 2009$ & $3 / 4 / 2009$ & $3 / 5 / 2009$ \\
\hline $\begin{array}{c}\text { SWEET B-P } \\
\text { Watts }\end{array}$ & $20 / 50$ & $30 / 60$ & $40 / 80$ & $40 / 90$ & $40 / 90$ & $50 / 90$ & $50 / 100$ & $50 / 100$ & $60 / 100$ & $60 / 100$ & $60 / 100$ \\
\hline $\mathrm{SpO}_{2}$ Base & 97 & 95 & 97 & 97 & 97 & 96 & 96 & 97 & 97 & 96 & 98 \\
\hline $\mathrm{SpO}_{2}$ Peak & 98 & 95 & 97 & 98 & 96 & 95 & 95 & 96 & 96 & 96 & 97 \\
\hline HR Base & 105 & 106 & 115 & 103 & 108 & 110 & 109 & 97 & 98 & 104 & 107 \\
\hline HR Peak & 120 & 121 & 125 & 119 & 122 & 124 & 124 & 104 & 106 & 107 & 115 \\
\hline $\mathrm{Rec} 6^{\prime} \mathrm{SpO}_{2}$ & 97 & 95 & 97 & 97 & 97 & 97 & 96 & 96 & 98 & 97 & 98 \\
\hline Rec 6' HR & 96 & 100 & 112 & 97 & 96 & 110 & 100 & 90 & 92 & 94 & 98 \\
\hline $\mathrm{VO}_{2} \mathrm{~L} / \mathrm{min}$ & 10 & 5 & 3 & 3 & 3 & 3 & 3 & 3 & 3 & 3 & 3 \\
\hline Sessions & 1 & 2 & 3 & 4 & 5 & 6 & 7 & 8 & 9 & 10 & 11 \\
\hline
\end{tabular}

SWEET: Square Wave Endurance Exercise Test during 45 minutes until the $11^{\text {th }}$ session (over 18 sessions); $\mathrm{B}=\mathrm{B} a \mathrm{se}$; $\mathrm{P}=\mathrm{Peak}$ : of the SWEET (watts/min) ; $\mathrm{SpO}_{2}$ : peripheral oxyhemoglobin saturation (\%). HR: Heart rate (beats/min); Rec: Recovery 6 minute after the SWEET; $\mathrm{VO}_{2}$ : oxygen therapy with face mask $(\mathrm{L} / \mathrm{min})$ to maintain $\mathrm{SpO}_{2}>94 \%$. The SWEET is subjectively well tolerated.

$(*)$ : dates are in American format: month/day/year

- In summary, hypoxemia was seen in all four muscle exercise tests. It should be noted that the reduction of $\mathrm{SpO}_{2}$ was less towards the end of the training because of the CDB and the learning of the ten first sessions of the SWEET $[9,13]$.

\section{Physical Therapeutics}

In Physical Therapy, the five stages of the Comprehensive Directed Breathing (CDB) [9] were used: [8,9].

1. The teaching and education of respiratory physiology, and normal nasal ventilation were explained as well as the interest and benefits of CDB $[8,9]$ with practical examples (diagrams, tables, spirometry, diaphragmatic brackets, maximum and spontaneous diaphragmatic mobility, [9] etc.) (30 min). Then, the physiotherapist repeated, at all respiratory rates: "Breathe through your nose and fill your lungs slowly and carefully, expanding your abdomen without moving either your shoulders or your chest; and now exhale the air slowly through your mouth, deflating your abdomen, and contracting your abdominal muscles until the last of the air is expelled, and again". The patient was instructed to match a breath volume displayed on an incentive spirometer (Voldyne, Sherwood Medical, St Louis, MO) by diaphragmatic breathing with feedback seated, supine, and side lying. The $\mathrm{CDB}$ was reinforced with verbal feedback to achieve a habitual pattern at rest and during the various proposed exercises walking, cycling, and climbing and descending a Two step stool and stairs. The visualization of VT (incentive spirometer) and other feedback repeated over 200 times during the first 90 min sessions, conditioned a new ventilatory pattern. Further assurance was provided by giving explanations about ventilatory efficiency, the new ventilatory rhythm, and the favorable physiologic outcomes of breathing retraining [8-13].

2. CDB application at rest (supine position, right and left lateral positions), sitting facing a mirror while breathing correctly (20 min) [8-11].

3. Exercises of upper extremity gymnastics, with weights in hand (1 kg in each hand), starting with $500 \mathrm{~g}$, then progressively increased up to $2 \mathrm{~kg}$ in each hand, but always accompanied by CDB [13] (20 min.)

4. The tests of conversation, reading a newspaper or watching television [13], always preventing physiological breakdown at the same time (CDB $30 \mathrm{~min})$.

5. The clinical control of the ventilatory frequency learned during all the muscular exercises mentioned (6 - MWT, 40W constant, TSST, upper extremity gymnastics and SWEET), but the tidal volume was controlled with an incentive spirometer only at rest [8].

Muscle training using $\boldsymbol{C D B}$ : Given the finding of hypoxemia in the four exercises performed, oxygen therapy with facial mask was established in all muscle training to maintain $\mathrm{SpO}_{2}$ above $94 \%$.

Training program: Six weeks with a total of 30 sessions were performed.

Simulating a 45-min intensive training session, the Square Wave Endurance exercise training (SWEET) is a "tailored" ergospirometry model for measurement of endurance and training. Preliminary measurements of Maximal Tolerated Power for 3 minute (MTP) i.e. maximum $\mathrm{VO}_{2}$, and the Ventilatory Anaerobic Threshold (VAT) [4] are required. It is expressed as Total Mechanical Work (TMW) in kJ/kg body weight. From the physiological point of view, the $\mathrm{VO}_{2}$ on the first minute represents about $65 \%$ of maximal constant exercise [8-11]. The SWEET is also be applied in transplanted cardiac patients $[14,15]$ or neumology [16].

The trend over the 45 minutes [17], without interruption and subjectively well supported, shows that in the base, ED increases at 15 minutes, then plateaus until the end $[11-13,17]$. No significant differences between minutes 15 and 30,15 and 45 or 30 and 45 were found. The evolution of the peak, unexpectedly, has a tendency to decrease over time, with ED at the end significantly lower than at the first 15 minutes $(\mathrm{P} \leq 0.001)$ [17]. To verify this, serum bicarbonate $\left(\mathrm{CO}_{3} \mathrm{H}^{-}\right)$, lactate and cortisol, were compared [9, 12] with rest, $\mathrm{VO}_{2}$ max and during the $45^{\text {th }}$ min of SWEET [19]. It appears that at rest, $\mathrm{CO}_{3} \mathrm{H}^{-}$was lowered in both exercise groups, which had significantly lower values compared to untrained subjects [19]. For lactate, the values were significantly higher at $\mathrm{VO}_{2}$ peak than during the SWEET [12]. With respect to cortisol, there was no difference between rest and $\mathrm{VO}_{2}$ Peak, while there were significantly higher values with SWEET than $\mathrm{VO}_{2}$ Peak $[4,8,12]$. Finally, the SWEET is the maximal training that maintains homeostasis during the full 45 minutes, the $\mathrm{pH}$ being in the normal range $[9,19]$. 
The distribution of the 90 minute sessions was as follows:

- SWEET [8, 9]: 3 sessions of 45 min each per week on alternate days,

- TSST [9-13]: A daily session of 15 minutes. The first two weeks were used as a warm-up before practicing the SWEET. The TSST $[10,11]$ session lasted 15 minutes; the test of walking 15 minutes, the test of gymnastics of upper limbs with weights 15 minutes and finally a 15 minute CDB practice [8]. Moreover, the patient could continue, (if he wishes but it is recommended), the training with a TSST at home.

The calculation of the physical work for 45 minutes of SWEET, was as follows: 1 Watt $(\mathrm{W})=1$ Joule $/ \mathrm{s}$ and $1 \mathrm{~min}$ : 60 seconds. Thus, before training: Base: $20 \mathrm{~W}$ x $36 \mathrm{~min}=720 \mathrm{~W}$. Peak: $9 \mathrm{~min} \times$ $50 \mathrm{~W}=450 \mathrm{w}$; Base + Peak $=720+450=1170 \mathrm{~W}$; now $1170 \mathrm{~W}$ x $60 \mathrm{~s}=70200 \mathrm{~J}$ or $70.2 \mathrm{KJ} / 100 \mathrm{Kg}$ or $0.72 \mathrm{KJ} / \mathrm{Kg}$. After training: 60W Base: $36 \mathrm{~min} \times 60 \mathrm{~W}=2160 \mathrm{~W}$; Peak: $9 \min \times 100 \mathrm{~W}=900 \mathrm{~W}$; Base + Peak $=2160+900=3060 \mathrm{~W} \times 60 \mathrm{~s}=183600 \mathrm{~J}$ or 183.6 $\mathrm{KJ} / 90 \mathrm{Kg}=2.04 \mathrm{KJ} / \mathrm{Kg}$; consequently, there was an increase of endurance of $+283 \%$ after 30 sessions of 45 minutes $[12,13]$.

The seventh session at the request of the patient was done without oxygen supply during the SWEET. The patient was able to maintain by the $\mathrm{CDB}$ a $\mathrm{SpO}_{2}$ of $94-95 \%$ until the $25^{\text {th }}$ minute, after which discreet hypoxia appeared, suggesting that SWEET can be performed for 45 minutes, with an outstanding ventilatory performance (Table 1).

In the second session the patient performed the TSST seven times for 15 minutes with oxygen therapy $\left(3 \mathrm{~L} \mathrm{VO}_{2} / \mathrm{min}\right.$ ) at the rate of 10 trips (up and down) per minute with a total of 300 effective steps, plus the energy spent on the descent of two steps, representing about a third of a single trip [10]. This allowed about a total of 390 steps in 15 minutes of continuous TSST sessions, well tolerated. In addition, there was hypoxia during the 6 - MWT and TSST before pulmonary rehabilitation (PR) but these could be performed without $\mathrm{O}_{2}$ after training, with normal $\mathrm{SpO}_{2}$. Moreover, similar training exercises were used for training subjects waiting before and after heart transplant [14-16].

Additionally, it should be noted that the maximum respiratory pressures (inspiratory and expiratory), measured after treatment were quite satisfactory and almost had normal values, taking into account the age and the pathology. With the improved physical performance (SWEET and TSST), the maximum respiratory pressures as $\mathrm{PI}_{\max }\left(78 \mathrm{~cm} \mathrm{H} \mathrm{H}_{2} \mathrm{O} ; 68 \%\right)$ and $\mathrm{PE}_{\max }\left(109 \mathrm{~cm} \mathrm{H}_{2} \mathrm{O}\right.$; $84 \%$ ) increased significantly. These results suggest that SWEETraining improved strength and endurance of both peripheral and respiratory muscles, as is commonly seen for other respiratory diagnoses $[1,3,5,6]$. In addition, the patient could continue, ad lib, the training with a TSST at home.

\section{Discussion}

For our patient, we obtained good muscular and ventilatory results with the different types of training using $\mathrm{CDB}$; this contrasts with what would be the usual prognosis and hopelessness for the severe obstructive ventilatory syndrome patient as seen in the literature [19-23]. More important is that after 6 weeks only of $\mathrm{CDB}$, exertional dyspnea was greatly reduced in the four exercise tests, without $\mathrm{O}_{2}$ supplementation, despite a very low FEV1. Normally, if FEV1 is low or very low [20-23], it is very hard to reduce the dyspnea and the asynchronisms. In our case, one possible explanation comes from the improvement of muscular, ventilatory and physical performance.

The results observed in our patient went in the right direction, which means that without significant changes in spirometric values, the physical performance improvement in the different exercises (walk, TSST and cycle ergometry tests) were significant, while dyspnea and leg pain decreased substantially. The patient found some real comfort and could walk three $\mathrm{Km}$ by a beach promenade without stopping, at his adapted acquired speed.

Another beneficial result observed in this patient from clinical point of view, was that he lost roughly $8 \mathrm{Kg}$ of total weight at the end of the full 30 sessions which also contributed and helped to reduce dyspnea during the treatment period $[27,28]$.

Additionally, we observed that the maximum respiratory pressures (inspiratory and expiratory), along with physical performance (SWEET and TSST), measured after the $11^{\text {th }}$ session of CDB treatment were quite satisfactory and almost had normal values, taking into account the age and the pathology. These results suggest that SWEETraining improved strength and endurance of both peripheral and respiratory muscles, as our team has seen it in other respiratory disorders $[1,3,5,6]$.

Traditional Diaphragmatic Breathing (TDB) has been reported to be ineffective in suppressing ventilatory asynchrony and reducing overbreathing. Some studies and reviews concluded that TDB worsens the coordination of chest wall motion as well as ventilatory activity and also increases respiratory work [19-23]. However, in this clinical case, the significant difference between before and after seem to stem from CDB efficacy and thus, from a more effective approach, to instituting breathing retraining. In fact, the new ventilatory pattern acquired by the patient, using $\mathrm{CDB}$, as seen by, a reduction in VE, RR and HR, contributed to significantly lower $\mathrm{VO}_{2}, \mathrm{VCO}_{2}$ and lactic acid production.. Lower breathing frequency and increased amplitude are critical to reverse overbreathing and improve respiratory efficiency [8-12]. Jones et al., [26] have shown a decrease in resting VO2 of 5\% after 10 min of diaphragmatic breathing, in patients with moderate to severe COPD and have attributed most of this change to the $14 \%$ decrease in respiratory rate. The increase in alveolar ventilation (VA/VE\% ratio), $\mathrm{SpO}_{2}$ and increase in peak inspiratory and expiratory flows indicated more efficient breathing. The modified ventilatory rhythm with $\mathrm{CDB}$ may favorably affect coordination of respiratory muscle recruitment $[8,9,11]$ which might in turn, explain increased efficiency of ventilation.

Our patient was obviously severe, with significant pulmonary emphysema, with very severe COPD, and great exertional dyspnea as well as advanced stage of the disease). The standard rehabilitation treatment given to him (classical TDB [19-23]), before he started our CDB protocol, was "simple", without any methodological basis, and not really effective. While after only six weeks of training with our SWEET and TWO STEP STOOL [10] techniques under $\mathrm{CDB}$, the results were significantly positive [27-30].

COPD patients are usually rehabilitated with a large bag of drugs (mostly corticoids and bronchodilators) given or prescribed by 
their physician (mostly pulmonologists) in one or more visits or medical exams, but unfortunately with no effective improvement on health status as was the case for our patient, not to mention some dangerous or unexpected (like fractures [27, 30]) side effects.

Oxygen therapy, during all the exercises, had to be provided when needed, to maintain normal $\mathrm{SpO}_{2}$ saturation.

\section{Conclusion}

We had a patient severely affected with COPD and significant dyspnea;

During 3 years after he was diagnosed, he only received in his local city, usual treatment, composed mostly of corticoid drugs, bronco-dilatators and modest (TDB) if any physiological rehabilitative help.

While he was followed by his local care team, he was advised to come to our rehabilitation department to try our alternative protocol of exercises (CDB) during 30 sessions of training (5 in each week) during 6 weeks minimum $[27,30]$.

The results were more than satisfactory, while not surprising us, as we had good results in our other studies [8-13, 19, 25, 27-30] where CDB was used.

Before he left, we advised him to return for a follow-up visit. Indeed, he came back 2 or 3 times and we retrained him with the same CDB protocol with almost the same good results.

Of course, if a patient respects all our recommendations of training (TSST) and stair climbing (BiSC) [25] at home, he would not need, or rarely need, to come back to the hospital.

We are still in contact with our patient and are glad of that because according to some authors $[29,30]$ the life survival of such severely affected patients of his age (almost 80 years) is only around 5 years [24].

Obviously, despite the good results observed, we advised our patient to return, on regular basis, for medical or rehabilitation monitoring of his healthy status.

\section{References}

[1]. Celli BR, Decramer M, Wedzicha JA, et al. An official American Thoracic Society/European Respiratory Society statement: research questions in chronic obstructive pulmonary disease. Am J Respir Crit Care Med. 2015 Apr 1;191(7):e4-e27. PubMed PMID: 25830527.

[2]. Casaburi R. Factors determining constant work rate exercise tolerance in COPD and their role in dictating the minimal clinically important difference in response to interventions. COPD. 2005 Mar;2(1):131-6. PubMed PMID: 17136973

[3]. Casaburi R, Patessio A, et al. Reductions in exercise lactic acidosis and ventilation as a result of exercise training in patients with obstructive lung disease. Am Rev Respir Dis. 1991 Jan;143(1):9-18. PubMed PMID: 1986689.

[4]. Gimenez M, Salinas W, Servera E, Kuntz C. VO2 max during progressive and constant bicycle exercise in sedentary men and women. Eur J Appl Physiol Occup Physiol. 1981;46(3):237-48. PubMed PMID: 7195806.

[5]. Minette A. Questionnaire of the European Community for Coal and Steel (ECSC) on respiratory symptoms. 1987--updating of the 1962 and 1967 questionnaires for studying chronic bronchitis and emphysema. Eur Respir J. 1989 Feb;2(2):165-77. PubMed PMID: 2703044.
[6]. Quajer P, Tammeling GJ, et al. Lung volumes and forced ventilatory flows. Report working party. Standardization of lung function test European Community for steel and Coal. Official statement of the European Respiratory Society. Eur Respir J Suppl. 1993 Mar;16:5-40. PubMed PMID: 8499054.

[7]. Borg GA. Psychophysical bases of perceived exertion. Med Sci Sports Exerc. 1982;14(5):377-81. PubMed PMID: 7154893.

[8]. Gimenez M, Servera E, Abril E, et al. Comprehensive directed breathing retraining improves exertional dyspnea for men with spirometry within normal limits. Am J Phys Med Rehabil. 2010 Feb;89(2):90-8. PubMed PMID: 19789433.

[9]. Gimenez M, Polu JM, Peńafiel M, Muńoz F. Physiopathological validation of Ventilation Directed at rest. Respiratory physiotherapy and SWEET raining. 2nd ed.In: Giménez M, Servera E, Vergara P, editors. Rehabilitation and Rehabilitation in Chronic Respiratory Pathology: Physiotherapy, training and respiratory care. Madrid: Pan American; 2004. p. 145-56.

[10]. Gimenez M, Saavedra P, Martin N, et al. Two-step stool aerobic training for smokers. Am J Phys Med Rehab. 2014 Jul 1;93(7):586-94.

[11]. Gimenez M, Lopez D, Abril E, et al. Comprehensive directed breathing and aerobic-anaerobic muscle training strongly improve a patient with severe COPD by pulmonary emphysema. Fisioterapia. 2010 Jan 1;32(5):236-42.

[12]. Gimenez M, Cereceda V, Teculescu D, Aug F, Laxenaire MC. Square-Wave Endurance Exercise Test (SWEET) for training and assessment in trained and untrained subjects. III: Effect on $\mathrm{VO}_{2}$ max and maximal ventilation. Eur J Appl Physiol Occup Physiol. 1982;49(3):379-87. PubMed PMID: 6890450.

[13]. Gimenez M, Abril E, Chabot F, Villemot JP, Mattei F, Polu JM. Strategies of physical training in ventilation of the patients before and after pulmonary transplant. Sports medicine. 1994; 68 (3): 146-56.

[14]. Mettauer B, Lampert E, Schnedecker B, et al. A short endurance training program increases the physical fitness of heart transplant recipients. Sci Sports. 1993 Jan 1;8(1):25-6.

[15]. Lampert E, Mettauer B, Lonsdorfer J. Effects of "niche" training on the late physical disability of cardiac transplant patients. Méd Sport. 1994; 68:14244.

[16]. Chabot F, Siat J, Gimenez M, Polu JM. Lung transplantation: Ventilation education and pre and post-operative muscular training, in Gimenez M, Servera E, Vergara P (eds): Prevention and Rehabilitation in chronic respiratory pathology: Physiotherapy, training and respiratory care.2nd ed. Madrid, Spain, Panamericana; 2004. p. 481-91.

[17]. Gimenez M, Saavedra P, Lantarón EM, et al. Exertional dyspnea Comparison in Four Maximal Training Exercises used in Pulmonary Rehabilitation: Continuous versus Bi-Level Responses in Time. Inter J Resp Dis Care \& Med. 2017 Jul 1;2(4):28-34.

[18]. Hermansen L, Osnes JB. Blood and muscle $\mathrm{pH}$ after maximal exercise in man. J Appl Physiol. 1972 Mar;32(3):304-8. PubMed PMID: 5010039.

[19]. Cahalin LP, Braga M, Matsuo Y, Hernandez ED. Efficacy of diaphragmatic breathing in persons with chronic obstructive pulmonary disease: a review of the literature. J Cardiopulm Rehabil. 2002 Jan-Feb;22(1):7-21. PubMed PMID: 11839992.

[20]. Dechman G, Wilson CR. Evidence underlying cardiopulmonary physical therapy in stable COPD. Cardiopulm Phys Ther. 2002 Jun 1;13(2):20.

[21]. Gosselink RA, Wagenaar RC, Rijswijk H, et al. Diaphragmatic breathing reduces efficiency of breathing in patients with chronic obstructive pulmonary disease. Am J Respir Crit Care Med. 1995 Apr;151(4):1136-42. PubMed PMID: 7697243.

[22]. Perez SB, Selleron B, Hotton R, Ferrali O, Sergysels R. Chest physiotherapy techniques-can they reduce hyperinflation. Rev Mal Respir. 2009 Dec;26(10):1107-17. PubMed PMID: 20032846.

[23]. Willeput R, Vachaudez JP, Lenders D, et al. Thoracoabdominal motion during chest physiotherapy in patients affected by chronic obstructive lung disease. Respiration. 1983 May-Jun;44(3):204-14. PubMed PMID: 6222449.

[24]. Oga T, Nishimura K, Tsukino M, et al. Exercise capacity deterioration in patients with COPD: longitudinal evaluation over 5 years. Chest. 2005 Jul;128(1):62-9. PubMed PMID: 16002917.

[25]. Gimenez M, Saavedra P, Lantarón EM, et al. Exertional dyspnea Comparison in Four Maximal Training Exercises used in Pulmonary Rehabilitation: Continuous versus Bi-Level Responses in Time. Inter J Resp Dis Care \& Med. 2017 Jul 1;2(4):28-34.

[26]. Jones AY, Dean E, Chow CC. Comparison of the oxygen cost of breathing exercises and spontaneous breathing in patients with stable chronic obstructive pulmonary disease. Phys Ther. 2003 May;83(5):424-31. PubMed PMID: 12718708.

[27]. Lorenzo N, Alonso MB, Martin N, et al. Patient with severe COPD, chronic respiratory failure and cardiorespiratory exacerbation treated with the CDBSWEETraining association. Fisioterapia. 2017.

[28]. Gimenez M, Pham QT, Uffholtz H. Prospective study on the long - term evolution of chronic bronchitis treated with or without controlled ventila- 
tion. International Colloquium of Cambo les Bains ;1977. p.317-332.

[29]. Gimenez M, Saavedra P, Martin N, et al. Bilevel exercise training and directed breathing relieves exertional dyspnea for male smokers. Am J Phys Med Rehabil. 2012 Oct;91(10):836-45. PubMed PMID: 22760109.
[30]. Gimenez M, Ûffholtz H, Pham QT, Sobradillo V. Ten years follow up with chronic obstructive lung disease submitted to a programmed of pulmonary rehabilitation. J R Soc Med. 1978 Jan;71(1):61-2. PubMed PMID: 633248. 